\title{
Pseudomonas aeruginosa: disseminação de resistência antimicrobiana em efluente hospitalar e água superficial
}

\author{
Pseudomonas aeruginosa: spread of antimicrobial resistance \\ in hospital effluent and surface water
}

\author{
Daiane Bopp Fuentefria ${ }^{1}$, Alessandra Einsfeld Ferreira ${ }^{1}$, \\ Tiago Gräf ${ }^{2}$ e Gertrudes Corção ${ }^{1,2}$
}

\begin{abstract}
RESUMO
O objetivo deste estudo foi comparar amostras de efluente do Hospital São Vicente de Paulo com amostras de água do Rio Passo Fundo, quanto ao perfil de susceptibilidade de isolados de Pseudomonas aeruginosa, para inferir sobre a presença de isolados de origem hospitalar em amostras de água superficial. A significância estatística entre os perfis de susceptibilidade das amostras foi testada por análise de variância e a comparação das amostras foi feita por contrastes de interesse. Foram identificados 198 isolados de Pseudomonas aeruginosa a partir das amostras analisadas. 0 fenótipo de multirresistência não foi observado nas amostras do Rio Passo Fundo, embora alguns isolados resistentes a carbapenêmicos tenham sido identificados, indicando a presença de contaminação com bactérias provenientes de um ambiente sob forte pressão seletiva. Diferenças significativas entre as amostras de água e efluente hospitalar foram observadas a partir da análise de variância por contrastes de interesse.
\end{abstract}

Palavras-chaves: Pseudomonas aeruginosa. Resistência bacteriana. Águas superficiais. Esgoto hospitalar.

\begin{abstract}
The aim of this study was to compare sewage samples from Hospital São Vicente de Paulo with water samples from the Passo Fundo river, with regard to the susceptibility profile of Pseudomonas aeruginosa isolates, in order to make inferences about the presence of strains of hospital origin in surface water samples. The statistical significance between the susceptibility profiles of the samples was tested using analysis of variance, and the samples were compared by means of contrasts of interest. One hundred and ninety-eight isolates of Pseudomonas aeruginosa were recovered from the samples analyzed. No phenotype for multiresistance was found in the samples from the Passo Fundo river, although some carbapenem-resistant isolates were identified, thereby indicating the presence of contamination with bacteria derived from an environment under strong selection pressure. Significant differences between the water and hospital effluent samples were observed, based on the analysis of variance by means of contrasts of interest.
\end{abstract}

Key-words: Pseudomonas aeruginosa. Bacterial resistance. Surface water. Hospital sewage.

Antibióticos e bactérias resistentes são descartados em grandes quantidades no ambiente ${ }^{6}$ como resultado do aumento e freqüente uso indiscriminado de antibióticos nas práticas médicas, veterinárias e agrárias. As águas dos rios são os principais receptores desses poluentes, uma vez que recebem os esgotos urbano, industrial e hospitalar. Os rios são considerados uma das principais fontes de água, direta ou indiretamente, para consumo humano e animal, portanto, esta poluição pode contribuir para a manutenção e até disseminação da resistência bacteriana $^{5}$. 0 esgoto hospitalar, especificamente, libera uma variedade de substâncias além de antibióticos, tais como fármacos, desinfetantes, anestésicos, metais pesados e drogas não metabolizadas por pacientes ${ }^{3}$. A ocorrência de ambientes fortemente seletivos para os microrganismos recuperados de efluentes hospitalares, gera um aumento da frequiência de genes de resistência bacteriana. Estudos têm demonstrado que efluentes hospitalares apresentam níveis mais elevados de bactérias entéricas resistentes a antimicrobianos do que efluentes derivados de outras fontes e, que a concentração de antimicrobianos na água que recebe o esgoto hospitalar também é superior, criando um ambiente com forte pressão seletiva ${ }^{10}$. Além disso, as precárias condições de saneamento básico e a falta de tratamento dos efluentes hospitalares podem estabelecer rotas de disseminação de bactérias multirresistentes ${ }^{113}$.

\footnotetext{
1. Programa de Pós-Graduação em Microbiologia Agrícola e do Ambiente, Universidade Federal do Rio Grande do Sul, Porto Alegre, RS. 2. Departamento de Microbiologia, Universidade Federal do Rio Grande do Sul, Porto Alegre, RS Órgãos financiadores: CAPES-PROF, PIBIC-CNPq

Endereço para correspondência: Prof̣ Gertrudes Corção. Depto de Microbiologia/ICBS/UFRGS. Rua Sarmento Leite 500. Cidade Baixa. 90050-170 Porto Alegre, RS.

Telefax: $55513308-4111$

e-mail: corcao@ufrgs.br

Recebido para publicação em 15/5/2008

Aceito em 14/07/2008
} 
A bactéria Pseudomonas aeruginosa é um dos principais microrganismos recuperados de efluentes hospitalares. É um patógeno nosocomial freqüente, responsável por infecções em diversos sítios do corpo humano, particularmente em pacientes imunocomprometidos. Está amplamente distribuída no ambiente e é capaz de persistir por longos períodos em ambientes adversos e desenvolver resistência a agentes antimicrobianos ${ }^{4}$. Além da característica intrínseca de apresentar baixo nível de sensibilidade aos antimicrobianos, diversos mecanismos de resistência têm sido identificados em Pseudomonas aeruginosa, como hiperexpressão de bombas de efluxo, produção de $\beta$-lactamases, perda ou expressão reduzida de proteínas de membrana externa $^{911}{ }^{12}$. Freqüentemente, isolados de Pseudomonas aeruginosa apresentam um amplo espectro de resistência, podendo ser resistentes a diferentes classes de agentes antimicrobianos, inclusive contra cefalosporinas de terceira e quarta gerações e carbapenêmicos (como imipenem e meropenem). Por estas razões, as infecções causadas por cepas de Pseudomonas aeruginosa multirresistentes estabelecem um substancial desafio para a terapia antimicrobiana, trazendo ao cenário atual a inevitável necessidade de identificar essas bactérias multirresistentes no efluente hospitalar e avaliar sua contribuição para a disseminação da resistência em amostras de água superficial.

Este estudo visa comparar o perfil de susceptibilidade entre amostras de efluente hospitalar e de água superficial, através de isolados de Pseudomonas aeruginosa provenientes das respectivas amostras, com o intuito de inferir sobre a presença de isolados de origem hospitalar em amostras de água superficial do Rio Passo Fundo.

\section{MATERIAL E MÉTODOS}

Amostras. Duas coletas foram realizadas, nos meses de outubro/2005 e março/2006, totalizando 12 amostras; seis de efluente hospitalar e seis de água superficial. As amostras de efluente foram coletadas do Hospital São Vicente de Paulo (HSVP), um hospital de 635 leitos, localizado em Passo Fundo, RS. As amostras de água superficial foram coletadas do Rio Passo Fundo em três pontos distintos: próximo à nascente do rio (Ponto A); à montante do descarte do efluente hospitalar (Ponto B) e à jusante do descarte do efluente hospitalar (Ponto C). Os Pontos D, E e F são amostras de efluente hospitalar e drenam diferentes setores hospitalares. Foram coletadas amostras de 11 de água superficial ou efluente hospitalar. Alíquotas de $100 \mathrm{~mL}$ foram filtradas em membranas de ésteres mistos de $0,45 \mu \mathrm{m}$ de porosidade. As membranas foram transferidas para 0 caldo seletivo asparagina e, posteriormente, alíquotas de $100 \mathrm{pl}$ dos tubos positivos foram transferidas para caldo acetamida e isoladas em ágar acetamida, para seleção de colônias características.

Identificação dos isolados de Pseudomonas aeruginosa. A identificação fenotípica foi realizada pela coloração de Gram e provas bioquímicas convencionais, como citocromo-oxidase, fermentação e oxidação da glicose, fermentação e oxidação da lactose, crescimento em caldo simples a $42^{\circ} \mathrm{C}$, motilidade, produção de $\mathrm{H}_{2} \mathrm{~S}$, redução de nitrato e produção de pioverdina. A identificação foi confirmada pela amplificação de um fragmento do $16 \mathrm{~S}$ rDNA de Pseudomonas aeruginosa.

Teste de susceptibilidade aos antimicrobianos. 0 perfil de susceptibilidade dos isolados de Pseudomonas aeruginosa foi determinado pela técnica de disco-difusão de acordo com as normas do Clinical Laboratory Standards Institute, 2005 ${ }^{6}$. Os seguintes

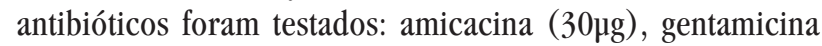

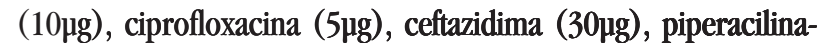

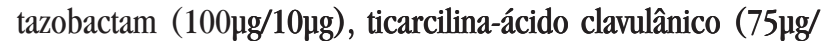

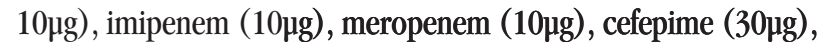
aztreonam $(30 \mu \mathrm{g})$ e polimixina B $(300 \mu \mathrm{g})^{4}$. Os isolados foram considerados multirresistentes quando apresentaram resistência a pelo menos quatro classes diferentes de antimicrobianos. A cepa Pseudomonas aeruginosa ATCC 27853 foi utilizada como controle de qualidade dos testes de susceptibilidade.

Análise estatística. Os perfis de susceptibilidade dos isolados de Pseudomonas aeruginosa nos diferentes pontos de coleta foram analisados, sendo a significância estatística entre as diferenças testada por meio de análise de variância por testes de aleatorização (com intervalo de confiança de 95\%). A hipótese nula $(\mathrm{Ho})$ formulada é de que não existe diferença entre os perfis de susceptibilidade dos grupos analisados. A comparação dos perfis de susceptibilidade foi feita por contrastes de interesse, a fim de comparar amostras de efluente hospitalar com amostras de água superficial. Os isolados de Pseudomonas aeruginosa foram considerados unidades amostrais e os antimicrobianos variáveis. Os dados originais foram transformados para dados semiquantitativos (sendo: $0=$ sensível; $1=$ intermediário e $2=$ resistente ao antimicrobiano testado) para cálculo de distância euclidiana entre unidades amostrais. A análise de variância foi realizada com auxílio do Programa Multiv (versão 242).

\section{RESULTADOS}

Foram identificados 198 isolados de Pseudomonas aeruginosa: 53 do Ponto B; 18 do Ponto C; 42 do Ponto D; 44 do Ponto E e 41 do Ponto F. Nenhum isolado de P. aeruginosa foi recuperado no Ponto A.

Os isolados de Pseudomonas aeruginosa do Ponto B apresentaram $77,3 \%$ de sensibilidade a todos os antimicrobianos testados, sendo que a principal resistência observada foi ao imipenem. No Ponto C, a taxa de sensibilidade a todos antimicrobianos testados foi de $44,4 \%$ e a resistência dos isolados foi verificada predominantemente frente ao meropenem, sendo $55,5 \%$ resistentes aos carbapenêmicos. Os isolados do Ponto D (efluente hospitalar) foram, na sua maioria $(80,9 \%)$ sensíveis a todos os antibióticos testados, com exceção de dois isolados que apresentaram resistência a imipenem, meropenem, cefepime e aztreonam. Os isolados de Pseudomonas aeruginosa do Ponto E também apresentaram alto $(65,9 \%)$ índice de susceptibilidade a todos os antimicrobianos testados, embora 34\% dos isolados tenham apresentado resistência aos carbapenêmicos. 0 Ponto $\mathrm{F}$ apresentou $73,2 \%$ de isolados resistentes a imipenem e/ou meropenem e $78 \%$ dos isolados com fenótipo de multirresistência (Tabela 1). 
Tabela 1 - Susceptibilidade dos isolados de Pseudomonas aeruginosa das amostras de água superficial (Pontos de coleta B e C) e efluente hospitalar (Pontos de coleta D, E e F).

\begin{tabular}{|c|c|c|c|c|c|}
\hline \multirow{3}{*}{$\begin{array}{l}\text { Dados gerais de } \\
\text { susceptibilidade }\end{array}$} & \multicolumn{5}{|c|}{ Pontos de coleta } \\
\hline & \multicolumn{5}{|c|}{$\mathrm{n}^{0}$ isolados Pseudomonas aeruginosa (\%) } \\
\hline & B $\left(n^{0}=53\right)$ & C $\left(n^{0}=18\right)$ & D $\left(\mathrm{n}^{\mathrm{0}}=42\right)$ & $\mathbf{E}\left(n^{0}=44\right)$ & $\mathbf{F}\left(\mathrm{n}^{0}=41\right)$ \\
\hline \multicolumn{6}{|l|}{ Isolados sensíveis a todos } \\
\hline os antimicrobianos testados & $41(77,3)$ & $8(44,4)$ & $34(80,9)$ & $29(65,9)$ & $4(9,7)$ \\
\hline \multicolumn{6}{|l|}{ Isolados resistentes a imipenem } \\
\hline e/ou meropenem & $12(22,6)$ & $10(55,5)$ & $6(14,3)$ & $15(34,0)$ & $30(73,2)$ \\
\hline Isolados multirresistentes & $0(0,0)$ & $0(0,0)$ & $2(4,8)$ & $0(0,0)$ & $32(78,0)$ \\
\hline
\end{tabular}

A análise de variância por aleatorização mostrou que o fator local de coleta apresenta diferenças significativas entre os grupos analisados $(\mathrm{p}<0,05)$. A Tabela 2 mostra que a comparação entre as amostras de água superficial e de efluente hospitalar apresentou diferenças significativas entre os contrastes analisados $(\mathrm{p}<0,05)$. Todos os contrastes analisados mostraram diferenças significativas $(\mathrm{p}<0,05)$.

Tabela 2 - Soma de Quadrados (Q) e Probabilidades (P) encontradas a partir da análise de variância analisando contrastes específicos entre amostras de água superficial (Pontos B e C) e esgoto hospitalar (Pontos D, E e F).

\begin{tabular}{lcc}
\hline Fator de variação: local & $\begin{array}{c}\text { Soma de } \\
\text { quadrados }(Q)\end{array}$ & $\mathrm{P}$ \\
\hline Entre grupos & 465,04 & 0,001 \\
Contrastes & & \\
$\quad$ Ponto B x Pontos DEF & 128,88 & 0,001 \\
$\quad$ Ponto C x Pontos DEF & 108,95 & 0,001 \\
$\quad$ Pontos BC x Pontos DEF & 180,70 & 0,001 \\
Dentro de grupos & 227,84 & \\
\hline Total & 692,87 & \\
\hline
\end{tabular}

\section{DISCUSSÃO}

Dentre os 198 isolados de Pseudomonas aeruginosa recuperados, 71 foram provenientes de água superficial. 0 ponto $\mathrm{A}$, próximo à nascente, não apresentou nenhum isolado de Pseudomonas aeruginosa, talvez por não receber efluente da Cidade de Passo Fundo e, portanto, não ser um local impactado. 0 ponto $\mathrm{C}$, à jusante do descarte do efluente hospitalar, apresentou menor número de isolados do que o ponto $\mathrm{B}$ (à montante); talvez, o Ponto B esteja recebendo outra fonte de contaminação, que tenha contribuindo para o maior número de isolados. Entretanto, quanto ao perfil de susceptibilidade, os isolados do Ponto B mostraram menor índice de resistência a carbapenêmicos, quando comparados aos isolados do Ponto C. Ao todo, 127 isolados foram recuperados das amostras do efluente hospitalar, sendo que o número de isolados de cada ponto não mostrou diferenças significativas.

Diversos estudos comprovam a presença de bactérias resistentes em água superficial No presente estudo, foi observada resistência a carbapenêmicos em amostras de água do Rio Passo Fundo (Tabela 1), embora o fenótipo de multirresistência não tenha sido observado. Além disso, a resistência ao imipenem encontrada em alguns isolados do Ponto B pode indicar uso de antibióticos carbapenêmicos na comunidade, uma vez que as águas do Ponto B não recebem efluente do HSVP, apenas efluente domiciliar e industrial. 0 uso doméstico de antibióticos pode estar exercendo pressão seletiva suficiente para permitir o aparecimento de isolados resistentes, aumentando o problema de resistência na comunidade ${ }^{2}$.

As amostras de efluente hospitalar, principalmente os pontos E e F, apresentaram alta porcentagem de isolados resistentes a carbapenêmicos e, no Ponto $\mathrm{F}$ especificamente, $78 \%$ de isolados multirresistentes, com resistência principalmente a gentamicina, ciprofloxacina, ceftazidima, piperacilina-tazobactam, ticarcilina-ácido clavulânico, imipenem e aztreonam. 0 Ponto $\mathrm{F}$ drena 0 maior número de enfermarias do HSVP, além de setores de coleta ambulatoriais e administrativos, assim, provavelmente a alta taxa de multirresistência tenha ocorrido, entre outros fatores, em função de água de banho desses pacientes e por ser o ambiente com menor número de Centros de Tratamento Intensivos (CTIs), provavelmente sujeito a menor controle de infecção.

Além de substâncias antimicrobianas e desinfetantes, bactérias resistentes também são excretadas por seres humanos e lançadas no efluente, podendo alcançar outros compartimentos ambientais ${ }^{8}$. A pressão seletiva exercida pelos antibióticos normalmente presentes no efluente hospitalar é um importante fator de seleção de bactérias resistentes, como as encontradas nos pontos E e F deste estudo. Por outro lado, existem muitas evidências de que a resistência antimicrobiana já esteja presente em ambientes naturais e que ocorra apenas a troca de genes entre bactérias ${ }^{10}$, fato que também pode estar ocorrendo entre os isolados do presente estudo, uma vez que os principais mecanismos de resistência aos antibióticos onde foi observada resistência (gentamicina, ciprofloxacina, ceftazidima, piperacilina-tazobactam, ticarcilina-ácido clavulânico, imipenem e aztreonam) são adquiridos e passíveis de transferência horizontal.

As diferenças significativas observadas a partir da análise de contrastes de interesse $(\mathrm{p}=0,001)$ ocorrem em função dos perfis de susceptibilidade dos isolados desses pontos. Quando amostras de água superficial (Pontos B e C) foram comparadas com amostras de efluente hospitalar (Pontos D, E e F) observou-se diferença significativa, que pode ser explicada, principalmente, pelo fato de que $78 \%$ dos isolados do ponto $\mathrm{F}$ apresentaram o fenótipo de multirresistência, enquanto que esse fenótipo não 
foi observado nos pontos B e C. Esses dados indicam que os isolados bacterianos presentes no efluente do HSVP apresentam um perfil de susceptibilidade diferente das recuperadas do rio Passo Fundo. Entretanto, o fato de nove isolados do Ponto C $\left(\mathrm{n}^{\circ}=18\right)$ apresentarem susceptibilidade reduzida ao meropenem não descarta a hipótese de que a contaminação dessas águas esteja ocorrendo, de alguma maneira, através do efluente hospitalar, pois o meropenem é um antibiótico de amplo espectro e de uso hospitalar restrito.

Nenhum isolado multirresistente de Pseudomonas aeruginosa foi encontrado nas amostras de água superficial do rio Passo Fundo e, diferenças significativas foram observadas entre os perfis de susceptibilidade dos isolados de água superficial e efluente hospitalar. Entretanto, um grau detectável de resistência pôde ser observado entre do Rio Passo Fundo, indicando uma possível contaminação por bactérias provenientes de um ambiente sob forte pressão seletiva, como é o ambiente hospitalar. Ambientes aquáticos contaminados podem constituir uma rota de disseminação de resistência bacteriana para a comunidade. Assim, reduzir a liberação de bactérias do ambiente hospitalar para a comunidade torna-se essencial para evitar o surgimento de reservatórios ambientais de resistência a antibióticos. Estudos de caracterização molecular adicionais devem revelar se existe ou não relação genética entre os isolados resistentes recuperados das amostras de água superficial e os isolados de efluente hospitalar, indicando uma possivel rota de contaminação neste ambiente.

\section{AGRADECIMENTOS}

Agradecemos à Comissão de Controle de Infecção Hospitalar do Hospital São Vicente de Paulo, pela aprovação das coletas de amostras de esgoto para este projeto.

\section{REFERÊNCIAS}

1. Baquero F. From pieces to patterns: evolutionary engineering in bacteril pathogens. Nature Reviews Microbiology 2: 510-518, 2004.

2. Clinical and Laboratory Standards Institute. Performance Standards for Antimicrobial Susceptibility Testing. In: Abstract of the $15^{\text {th }}$ Informational Supplement. Clinical and Laboratory Standards Institute document. M100-S15 (ISBN 1-56238-556-9), Wayne, Pennsylvania, USA p.38-39, 2005.

3. Emmanuel E, Perrodin Y, Keck G, Blanchard JM, Vermande P. Ecotoxicological risk assessment of hospital wastewater: a proposed framework for raw effluents discharging into urban sewer network. Journal of Hazardous Materials A117: 1-11, 2005.

4. Gales AC, Reis AO, Jones RN. Contemporary assessment of antimicrobial susceptibility testing methods for polymixyn B and colistin: review of available interpretative criteria and quality control guidelines. Journal of Clinical Microbiology 39: 183-190, 2001.

5. Goni-Urriza M, Capdepuy M, Arpin C, Raymond N, Caumette, P, Quentin C. Impact of an urban effluent on antibiotic resistance of riverine Enterobacteriaceae and Aeromonas spp. Applied and Environmental Microbiology 66: 125-132, 2000.

6. Halling-Sorensen B, Nors Nielsen S, Lanzky PF, Ingerslev F, Holten Lützheft HC, Jorgensen SE. Occurrence, fate and effects of pharmaceutical substances in the environment - a review. Chemosphere 36: 357-393, 1998.

7. Kümmerer K. The significance of antibiotics in the environment. Journal of Antimicrobial Chemoterapy 52: 5-7, 2001.

8. Kümmerer K. Resistance in the environment. Journal of Antimicrobial Chemoterapy 54: 311-320, 2004.

9. Li XZ, Barre N, Poole K. Influence of the MexA-MexB-oprM multidrug efflux system on expression of the MexC-MexD-oprJ and MexE-MexF-oprN multidrug efflux systems in Pseudomonas aeruginosa. Journal of Antimicrobial and Chemotherapy 46:885-893, 2000.

10. Linton KB, Richmond MH, Bevan R, Gillespie WA. Antibiotic resistance and R factors in coliform bacilli isolated from hospital and domestic sewage. Journal of Medical Microbiology 7: 91-103, 1974.

11. Livermore DM. Multiple mechanisms of antimicrobial resistance in Pseudomonas aeruginosa: our worst nightmare? Clinical Infectious Disease 34:634-640, 2002.

12. Masuda N, Ohya S. Cross-resistance to meropenem, cephems, and quinolones in Pseudomonas aeruginosa. Antimicrobial and Agents Chemotherapy 36: 1847-1851, 1992.

13. Muela A, Pocino I, Arana J, Justo JI, Iriberri J, Barcina I. Effects of growth phase and parental cell survival in river water on plasmid transfer between Escherichia coli strains. Applied and Environmental Microbiology 60: 4273-4278, 1994. 